\section{Polymyalgia Rheumatica and Giant Cell Arteritis}

Bhaskar Dasgupta and Christian Dejaco, eds. Oxford: Oxford University Press, 2016, 192 pages, $\$ 42.50$ US

This first edition of Polymyalgia Rheumatica and Giant Cell Arteritis is a welcome addition to the pocket book family. The authors' goals were to provide quick and practical updated information on the epidemiology, diagnosis, and management of polymyalgia rheumatica (PMR) and giant cell arteritis (GCA).

The book is separated into 4 sections. Section 1 describes the epidemiology, pathogenesis, and clinical presentation of PMR and GCA. Most of the epidemiologic data presented are European, with little information on North America and Asia. The chapter on pathogenesis is reader-friendly and contains many pictures and diagrams illustrating the pathophysiology and immune responses involved in PMR and GCA. Section 2 describes the diagnostic investigation and provides a useful summary on the use of ultrasound, magnetic resonance imaging, and positron emission tomography, with their advantages and pitfalls in GCA and PMR. Section 3 provides current guidelines for treatment with glucocorticoids and explores the role of steroid-sparing agents. The disease-modifying antirheumatic drugs and biological agents are presented in an easy-to-read table including their suggested indication and evidence. Unfortunately, little information is given regarding the use of low-dose aspirin to reduce visual loss and cerebrovascular complications in GCA; it will be up to the reader to review the literature and rationale behind it. Section 4 describes the prognosis and socioeconomic burden associated with PMR and GCA while exploring the necessity of qualitative research to better understand patients having these conditions.

Despite minor faults mentioned above, the authors reached their goal of providing a practical guide for those seeking information on diagnosis and management of PMR and GCA. The recommended audience includes rheumatologists, rheumatology trainees, general practitioners, and other professionals caring for patients with PMR and GCA.

JEAN-PAUL MAKHZOUM, MD, FRCP(C), Fellowship in Systemic Autoimmune Rheumatic Disease, University of Toronto, Toronto, Ontario, Canada. Address correspondence to Dr. J.P. Makhzoum,

7 Tullis Drive Upper, Toronto, Ontario M4S 2E2, Canada. E-mail: jeanpaul.makhzoum@mail.utoronto.ca

J Rheumatol 2016;43:11; doi:10.3899/jrheum.160996 